\title{
A spatially explicit assessment of middle and low voltage grid requirements in Bavaria until 2050
}

\author{
Luis Ramirez Camargo ${ }^{1 凶}$ - Jane Wuth ${ }^{1}-$ Markus Biberacher $^{2}$ - Wolfgang Dorner ${ }^{1}$ \\ ${ }^{1}$ Institute for Applied Informatics, Technologie Campus Freyung, Technische Hochschule Deggendorf, Freyung, Germany \\ ${ }^{2}$ Research Studio iSPACE, Salzburg, Austria \\ ${ }^{\otimes}$ luis.ramirez-camargo@th-deg.de
}

\begin{abstract}
The energy transition towards high shares of renewables and the continued urbanization process have a direct and strong impact on the shape and characteristics of the electricity transmission and distribution systems. At the continental and national scale, improved high voltage grids should allow the transmission and balance of electricity from hot-spots of variable renewable energy generation installations to demand centres. At the regional and municipal scale, the medium and low voltage grids should be capable of bringing sufficient electricity to users and allow the integration of distributed renewable generation installations. While data on the transmission systems is widely available, spatial and attribute data of the medium and mainly the low voltage grids are scarce. Additionally, while there are plenty of studies on the requirements of the grid to allow the energy transition, there is very little information on the necessary transformation of the grid due to changes generated by the expected urbanization process. This study relies on a data set that estimates the topology of the medium and low voltage grids of Bavaria (Germany) as well as data from the LUISA territorial modelling platform of the European Commission to calculate key figures of grid requirements depending on population and land use for the current case and the decades to come. Typologies of grid requirements are proposed based on a statistical analysis of population and land use data of each square kilometre of the federal state. These typologies are extrapolated to changes in the structure of settlements that are expected in the years 2030 and 2050. Results are presented using maps with expected absolute values of grid requirements and their temporal changes for each square kilometre of the project area. Grid requirements are expected to increase in cities and to be reduced in most of the rural areas. The largest changes are expected to take place in the suburbs of the major cities.
\end{abstract}

\section{Highlights for public administration, management and planning:}

- Medium and low voltage grid shapes and lengths are estimated for the entire federal state of Bavaria, Germany.

- On average, distribution grid length requirements per person are between 13 and 16 times larger in rural regions than in city centres.

- While city centres and suburbs expect an increase in grid requirements, the total grid length of Bavaria is expected to decrease in the near future.

- Suburbs of large cities are not only expected to change steadily but also to show the largest changes in grid length requirements until 2050.

\section{Introduction}

Todays most anticipated change in the energy system is the transition to renewable energy generation sources and its expected effect on the elec-

\section{Keywords}

Urbanization, Low voltage grid, Medium voltage grid GIS,

Energy transition

Received:

04 July 2019

Received in revised form: 04 October 2019

Accepted:

17 October 2019 
many will be a main photovoltaic electricity provider as here solar radiation and landscapes are especially favourable. Due to large urbanized regions and electricity intensive industries, nevertheless, Bavaria consuming more electricity than they can produce. Not yet well researched, are impacts on the power grid due to changes in the structure and size of settlements and especially rural depopulation processes. Especially rural border regions in Bavaria are suffering from rural exodus and shrinking economic and infrastructural possibilities. Both topics, the energy transition as well as the structural changes, have in common that they are directly and strongly impacting the shape and characteristics of the electricity transmission and distribution systems. Nevertheless, what is currently considered most in research, is the transition to renewable energies, the emerging instabilities that could arise through it and the needed stabilization measures to secure the functioning of the grid. Effects of structural changes in settlements and rural depopulation are not as much considered as they are of concern. Results emerging from depopulation in rural regions are, growing cities which already today have difficulties of being supplied with sufficient energy. Especially when considering the needed space for installations and the resulting distance to the next building or city of renewable energy sites as well as transmission losses over the resulting long distances that the electricity has to be carried, the electricity supply to cities may be severely impacted by the ongoing structural settlement changes.

Grid optimization is therefore indispensable at the continental and national scale as well as the regional and municipal level. Considering the former case, high voltage grids need to be extended to allow the transmission and balance of electricity from hot-spots of variable renewable energy generation installations to demand centres. In the latter, the distribution system consisting of a medium voltage grid (primary distribution system with voltages between $4 \mathrm{kV}$ and $35 \mathrm{kV}$ ) and low voltage grid (secondary distribution with voltages between $220 \mathrm{~V}$ and $240 \mathrm{~V}$ for the European case) should be capable of delivering sufficient electricity to users and simultaneously allow the integration of distributed renewable generation installations (Short 2004). While data for analysing the transmission systems is widely available, spatial and attribute data of the medium and mainly the low voltage grids are scarce. These are necessary for detailed studies on the integration of distributed generation installations. Their availability for large study areas would enable research on the definition of op- timal settlement structures for the development of e.g. energy communities with very high or $100 \%$ renewable energy supply. To be able to perform research on the mentioned topics, the different power grids therefore need to be calculated. Currently, studies propose diverse ways how to calculate the grid and its hosting capacities among other important factors. Alturki, Khodaei, Paaso, \& Bahramirad (2018) for instance summarize research in the field of optimizing hosting capacities and developing a new method with focus on the grid instabilities that may arise due to a rising number of prosumers. The topic of hosting capacities is well researched and covers the question of the impact on and capabilities of the grid, when considering rising importance of renewables. Guntaka \& Myler (2014) go further by applying krigin and linear least square regressions in order to obtain power factor amounts at critical nodes without ground connections. This calculation provides a part of the basis to calculate the overall transmission grids efficiency, however no spatially georeferenced data was gathered but the calculations rather took place in a laboratory environment. Hess et al. (2018) take a different look at the problem. Their hypothesis is that with the introduction of renewable energies and multiple new generation points within the grid, major grid expansions will take place, whose costs need to be considered when modelling the energy system. Their model focuses on the node-internal transmissions and spatial distribution of the electricity grid. Nevertheless, also these authors focus solely on the transmission grid, and only partially include the distribution grid, which can be validated with the existing official data.

Independent of the study focus, effects resulting from changes in settlement structures or renewable energies integration measures, urban energy modelling is of major importance. Keirstead et al. (2012) draw the conclusion that a connection between different topics, such as grid lengths and population developments or the correlation of land use and grid requirements should be further researched. To the authors' knowledge, there is until today no article addressing these topics.

Existent research today is rather focused on the topic of integrating renewable energies without destabilizing the grid. Besides simulations of PV and battery installations at household and on communal level (Marczinkowski \& Østergaard 2018) or energy systems modelling for future situations (Østergaard et al. 2015), methods such as distributed generation (Ramirez Camargo \& Stoeglehner 2018) or "Vehicle-to-Grid" (V2G) services (Child et al. 2018; Prasomthong et al. 2014) 


\section{Sciendo}

have won momentum in scientific literature. What these studies have in common is that they focus rather on the problems in combination with renewable energies than the impacts that may arise due to the urbanization and rural depopulation streams. Up until the 1970s, the urbanization (people moving to the city centres) and thereafter the suburbanization (people moving into the cities' catchment area) increased the population and size of cities in developed countries. Afterwards, researchers realized that a counterurbanization trend was rising, meaning that people started moving away from city centres (Antrop 2004). Nevertheless, this did not mean that very rural regions, further away from cities were benefiting from this trend. In the 1980's, most of Bavaria was categorized as rural regions with unfavourable structures to expand existing rural settlements (Stiens 1986). Also based on the LUISA territorial modelling platform of the EU Science Hub, the estimations until 2050 do not seem to be more favourable for Bavarian rural regions (Barbosa et al. 2015). The goal of this article is therefore to motivate a discussion and research concerning the impacts of urbanization on the medium and low voltage grid length requirements in Bavaria. The method relies on a data set that estimates the topology of the medium and low voltage grids of Bavaria (Germany) as well as on data from the LUISA territorial modelling platform of the European Commission to calculate grid requirements for the years 2030 and 2050. Two alternative paths are proposed to carry out these calculations. The first is based on population data and the second on land use data. Grid length typologies are created based on population thresholds or land use types for 2010. The same typologies are afterwards used to extrapolate grid requirements for the future, which follow the population and land use changes of the LUISA data set. The future estimations are therefore in conformity with the forecasts utilized by the European Commission. The rest of this paper is structured as follows: the next chapter describes the data used and the methodology applied, whose results are presented and discussed in the subsequent section. Conclusions and proposals for further research are drawn in Section 4.

\section{Data and methodology}

Two alternative paths are presented to estimate medium and low voltage grid requirements in 2030 and 2050 for the state of Bavaria in Germany. The necessary data to calculate these grid requirements are on the one side population and land use data from the LUISA Territorial Modelling Platform, and on the other side geographically referenced data of the current medium and low voltage grids in Bavaria. The population and land use data of the LUISA Territorial Modelling Platform are provided by the European Science Hub (the science and knowledge platform of the European Commission) and are the result of efforts of the European Commission to provide a dataset that presents expected developments of the continent in terms of land use and population distribution changes until 2050 (Barbosa et al. 2015). The data set includes information of all European Union member states for the years 2010, 2020, 2030, 2040 and 2050 and is provided at no cost in Geotiff format in EPSG 3035. The population data set used in the present study contains the amount of inhabitants in a spatial resolution of $1 \mathrm{~km}^{2}$, later referred to as one pixel. The land use data set presents 9 different classes of land use in the same resolution. The land use classes include agricultural, urban fabric, industrial/commercial, infrastructure, Forest, Natural land, urban green leisure, wetlands and water bodies.

The geographically referenced data of the current medium and low voltage grids in the state of Bavaria are obtained using a method that was developed and adjusted with Austrian grid information. In order to bottom-up identify the topology of a relevant grid infrastructure starting from the distribution grid, a spatial discrete identification of possible objects to be connected to the grid has been performed. It is mainly based on objects outlined in OpenStreetMap (OSM) (OpenStreetMap, o.J.). Furthermore, the existing road infrastructure (also from OSM) has been considered as an indication for possible distribution grid nodes and topologies. The approach to identify the (semi-)optimal topology for the relevant grid infrastructure uses the principle of a minimum spanning tree. It is realized by using the greedy algorithm of Kruskal (Kruskal 1956). It identifies among a sorted list of weighted edges the relevant subset of edges which represent a minimum spanning tree for all nodes to be connected. 


\section{sciendo}

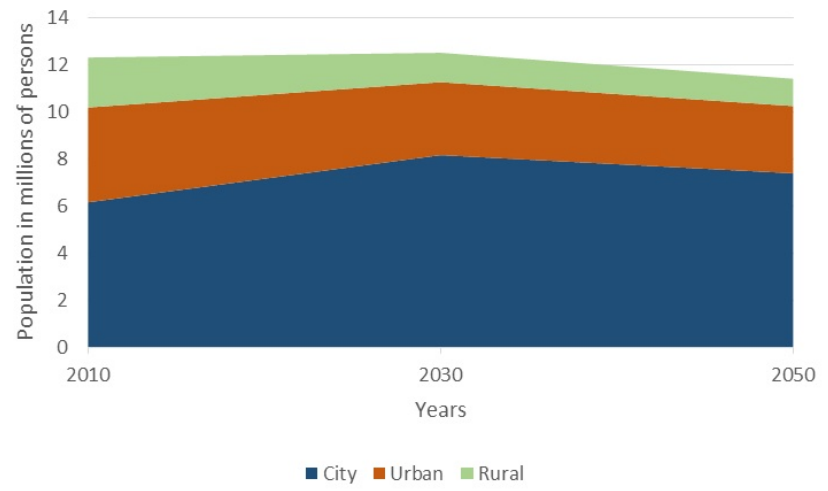

Fig. 1 Population development for Bavaria from 2010 until 2050 based on LUISA population data differentiated by city, urban and rural area types. Source: the authors using LUISA population data

The algorithm has been implemented in $\mathrm{C}++$ and was extended by further assumptions on limitations regarding voltage stability and maximum transformer capacities in low voltage clusters. The approach identifies an optimal clustering for low-voltage clusters and corresponding transformer locations. In a further step, the identification of an optimal middle-voltage topology is calculated with the same approach. It is based on the iden- tification of possible middle-voltage edges by routing between the identified transformer locations. A validation of the approach has been performed in an Austrian case study (not public) with real grid data and the achieved results showed a high correlation with the real data. It showed especially, that the approach covers well the varying situations between urban and rural areas.

These grid datasets are rasterized into $10 \mathrm{~m} \mathrm{x} 10 \mathrm{~m}$ maps of medium and low voltage grids. To fit the extent and resolution of the LUISA datasets covering Bavaria, the grid dataset was resampled. Every pixel of the grid maps, represents now the length of the low and medium voltage grid respectively in the same resolution as the LUISA datasets.

The first option considered to estimate the future grid requirements is entirely based on the LUISA population dataset. Based on the thresholds of population densities proposed by Dijkstra and Poelman (2014) three grid typologies to define high population density (city centres), urban and rural areas in the European Union are generated. Rural areas consist of less than 300 inhabitants per square kilometre, urban areas consider regions between 300 and 1500 inhabitants

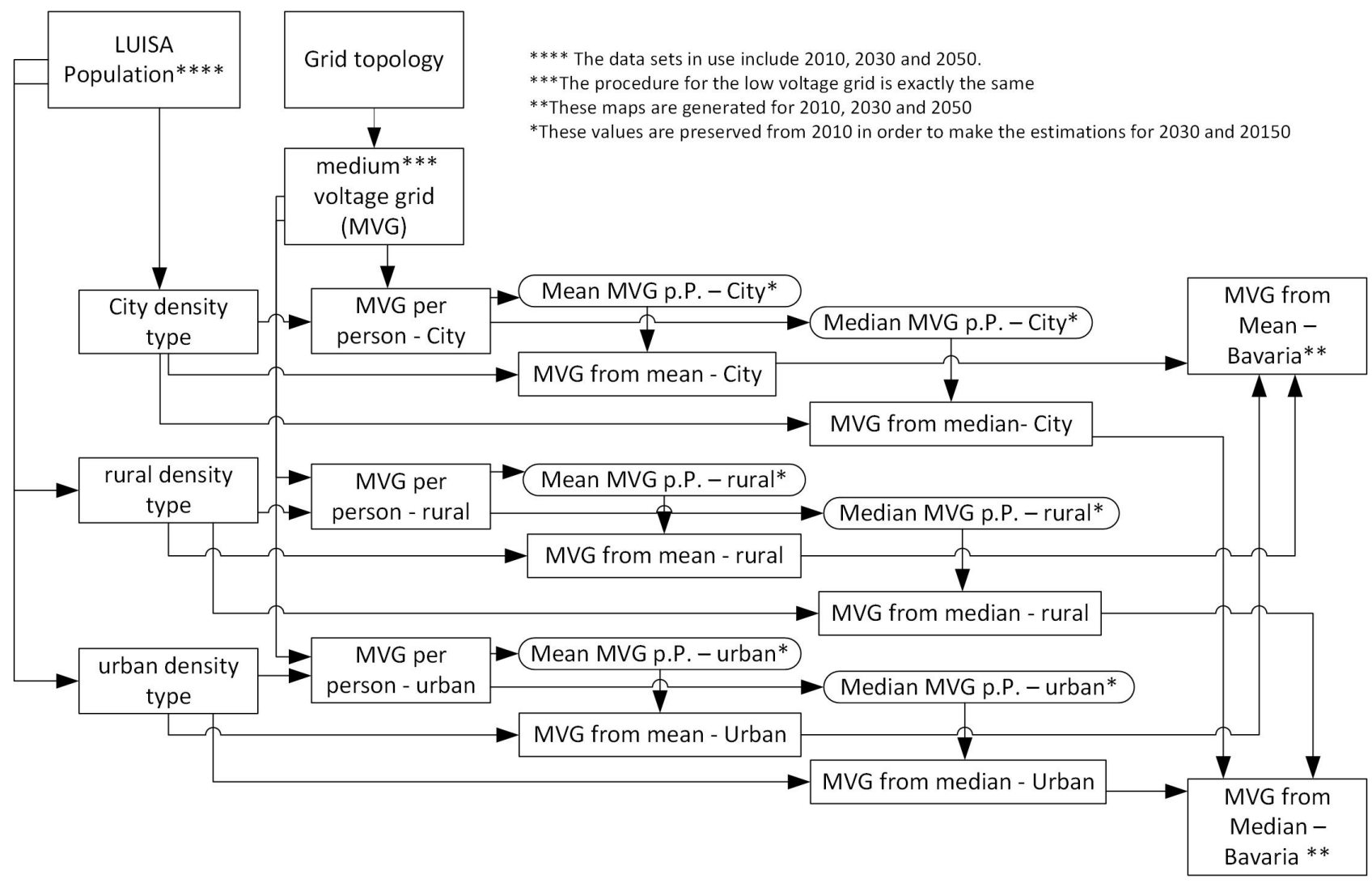

Fig. 2 Schematic description of the procedure to estimate grid length for 2010, 2030, 2050 based on population data: Source: authors 
and city centres cover square kilometres consisting of more than 1500 inhabitants. These thresholds are used to classify the population maps for 2010, 2030 and 2050 and to generate masks for every type of population density (city, urban, rural). Fig. 1 shows the population development of Bavaria based on this data and classification.

The grid density per person is calculated for each pixel dividing the calculated grid maps (low and medium voltage) by the population map of 2010 . The resulting masks of population density types are used to calculate descriptive statistics of grid density per person for each one of the population density types. Mean and median values of these statistics are multiplied (separately) by the population data of the pixels corresponding to each one of the population density types. By patching together the resulting maps for city, urban and rural grid length, it is possible to produce grid length maps covering the entire state of Bavaria. Descriptive statistics of these maps, one based on the mean value of the grid density per person for each one of the population density types and the other one based on the median, are calculated and compared to the descriptive statistics of the original grid maps. The map statistically considered closest to the original is used as the reference map. The values of grid length per person used for each population density type in the reference map, are employed to estimate grid lengths for the years 2030 and 2050. This is done in the same way as before when calculating the reference map i.e. the grid length per person for each population density type is multiplied by the pixels that belong to the certain density type. The three resulting grid length maps for city, urban and rural types are patched together and an estimation map is produced for the years 2030 and 2050. This procedure is applied to the medium and the low voltage grids individually. A schematic description of the procedure is presented in Fig. 2.

A second option considered to estimate future grid requirements is based on the LUISA land use data. Instead of using the population statistics to create a population density based cluster, the typologies correspond to the different land uses provided in the LUISA data set. Masks are generated for the agricultural, industrial, infrastructure and urban uses as well as for a fifth use type called "others", which summarizes the remaining five land use types of the LUISA dataset. Apart from the typologies, the rest of the procedure remains equal to the first one. Grid length maps are calculated for the years 2010, 2030 and 2050 for low and medium voltage grids.

\section{Results and discussion}

The methodology presented in the previous chapter was implemented using gdal/ogr (GDAL Development Team, 2016) and GRASSGIS 7 (Neteler \& Mitasova 2008). The mean and median values of grid length per person for each typology are summarized in Tables 1 and 2. A common denominator of these statistics is that mean and median values are relatively close to each other for the population density types city and urban as well as the land use type urban. The values in these categories are well distributed around the mean and both statistics are good candidates to be used as predictors of grid length per person. In contrast, there are strong differences between the mean and median grid length per person in the population density type rural and all other land use types but urban.

Table 1 Statistics of the population density types for medium and low voltage grid in 2010

\begin{tabular}{|c|c|c|c|c|c|c|}
\hline \multirow[b]{2}{*}{ Statistic } & \multicolumn{3}{|c|}{$\begin{array}{l}\text { Medium Voltage } \\
\text { Grid length per } \\
\text { person* }\end{array}$} & \multicolumn{3}{|c|}{$\begin{array}{l}\text { Low Voltage } \\
\text { Grid length } \\
\text { per person* }\end{array}$} \\
\hline & Rural & Urban & City & Rural & Urban & City \\
\hline Mean & 70.22 & 3.40 & 1.62 & 230.68 & 16.75 & 7.83 \\
\hline Median & 24.39 & 2.90 & 1.48 & 92.34 & 14.13 & 7.03 \\
\hline
\end{tabular}

* in each $\mathrm{km}^{2}(\mathrm{~m})$ based on population density types; source: authors

The consequences of the differences in mean and median grid length values of the typologies are reflected in the statistics of the grid length maps estimated for 2010, which are presented in Tables 3 and 4 . Only in the estimation where the median value of the grid length of each population density category is used, the mean and the sum of the grid length are similar to the original grid maps. In the case of the medium voltage grids, further statistics such as the standard deviation and the values at the 3rd quartile and 99.9 percentile are similar to the original dataset. For the other three calculation alternatives, even basic statistics such as mean and sum are considerably different to the ones of the original maps. The estimated maps using land use typologies perform worse than the maps generated using mean length values of the population density typologies. A reason why the grid length per pixel is not well depicted by land use typologies is because they do not correlate with each other. On the other hand, when considering population, this is a different case. Assuming that the needs of citizens for electricity are similar between ru- 
Table 2 Statistics of the land use types for medium and low voltage grids in 2010

\begin{tabular}{|c|c|c|c|c|c|c|c|c|c|c|}
\hline & \multicolumn{5}{|c|}{$\begin{array}{l}\text { Medium Voltage } \\
\text { Grid length* }\end{array}$} & \multicolumn{5}{|c|}{$\begin{array}{l}\text { Low Voltage } \\
\text { Grid length* }\end{array}$} \\
\hline Statistic & Agric. & Ind. & Infra. & others & urban & Agric. & Ind. & Infra. & others & urban \\
\hline Mean & 52.50 & 31.49 & 97.87 & 93.03 & 2.38 & 180.5 & 125.0 & 295.0 & 295.4 & 12.6 \\
\hline Median & 16.30 & 6.28 & 19.88 & 34.31 & 1.83 & 67.8 & 25.9 & 72.9 & 131.2 & 10.2 \\
\hline
\end{tabular}

* per person in each $\mathrm{km}^{2}(\mathrm{~m})$ based on land use types; source: authors

ral and urban regions, the size of the population has a direct link to the need for grid and junction boxes. However, land use typologies apparently do not provide such a strong connection to the grid requirements, potentially because the individual land use types do not homogeneously indicate how much electricity is needed. In Industry for example, the amount of electricity or grid length needed is very closely interlinked with the type of product or service that is provided there. In the Land Use types Infrastructure and Urban, the grid length would vary because it might be decisive how densely populated the pixel under consideration is. Consequently, it was realized that the land use typologies cannot be used as grid length denominators for further calculations. Fig. 3 displays the medium voltage grid length for each pixel in Bavaria. The very left map presents the length of today's grid not yet clustered into different population sizes or land use types. It is apparent that the largely urbanized regions, Munich in the south and Nuremberg more in the north, include more grid length per pixel than regions in the very east or west of the federal state. The regions at the Bavarian borders are very rural for the most parts and less distribution grid is necessary to supply everyone with sufficient electricity. The middle and right maps show the two different approaches of calculating grid requirements depending on population sizes per pixel. The map in the middle uses the mean grid length per person for each population density category defined above (city, urban, rural). The same approach was applied to the map on the right, only instead of the mean grid length per person, the median grid length per person was used.

Comparing the two calculated maps it is apparent, that when considering the mean length per pixel, the result does not seem to be clustered compared to results using the median grid length. The estimated mean length of the medium voltage grid using the mean grid length for the calculation, is 2439 $\mathrm{m}$ per pixel. Comparing this to the actual mean length of $1267 \mathrm{~m}$ in the original map, it seems that the mean length per person does not depict reality in a good way. Therefore, further calculations were executed using the median grid length per person and cluster. Here, the mean length per pixel is $1025 \mathrm{~m}$, which is very close to the original map (1 $267 \mathrm{~m})$. This situation is the same for the low voltage grid. The resulting mean grid length in $\mathrm{m}$ per pixel of the map estimated using the median is $4181.76 \mathrm{~m}$, while the mean grid length of the original map is 4008.7. In fact, the low voltage grid length values for rural and urban areas per person calculated using the median, $92.34 \mathrm{~m}$ and $14.13 \mathrm{~m}$ respectively, is very close to values reported by specialized literature. Short (2004) shows that typical

Table 3 Statistics of the original and estimated low voltage grid length maps

\begin{tabular}{|c|c|c|c|c|c|}
\hline $\begin{array}{c}\text { Low Voltage } \\
\text { Grid Descriptives }\end{array}$ & $\begin{array}{c}\text { Original } \\
\text { Grid Length }\end{array}$ & \multicolumn{2}{|c|}{$\begin{array}{l}\text { Grid Estimated Using } \\
\text { Population Density Typologies }\end{array}$} & \multicolumn{2}{|c|}{$\begin{array}{l}\text { Grid Estimated Using } \\
\text { Land Use Typologies }\end{array}$} \\
\hline Subject & Length/km² & $\begin{array}{c}\text { Estimated } \\
\text { mean } 2010 \\
\text { Length } / \mathrm{km}^{2}\end{array}$ & $\begin{array}{c}\text { Estimated } \\
\text { median } 2010 \\
\text { Length } / \mathrm{km}^{2}\end{array}$ & $\begin{array}{c}\text { Estimated } \\
\text { mean } 2010 \\
\text { Length } / \mathrm{km}^{2}\end{array}$ & $\begin{array}{c}\text { Estimated } \\
\text { median } 2010 \\
\text { Length } / \mathrm{km}^{2}\end{array}$ \\
\hline Maximum & 43574.2 & 104339 & 93737.3 & 2.19528 & 975222 \\
\hline Sum & 286966628.8 & 604868471.5 & 296010002.5 & 1124679992.5 & 468972081 \\
\hline Median & 2523.78 & 2768.26 & 1108.1 & 2527.35 & 1017.06 \\
\hline 3rd quartile & 4234.88 & 11534.4 & 5725.19 & 12407.7 & 5249.48 \\
\hline 99.9 Percentile & 36614.1 & 68745 & 55156.1 & 614182 & 243313 \\
\hline
\end{tabular}

source: authors 


\section{sciendo}

Table 4 Statistics of the original and estimated medium voltage grid length maps

\begin{tabular}{|c|c|c|c|c|c|}
\hline $\begin{array}{l}\text { Medium Voltage } \\
\text { Grid Descriptives }\end{array}$ & $\begin{array}{l}\text { Original } \\
\text { Grid Length }\end{array}$ & \multicolumn{2}{|c|}{$\begin{array}{l}\text { Grid Estimated Using } \\
\text { Population Density Typologies }\end{array}$} & \multicolumn{2}{|c|}{$\begin{array}{l}\text { Grid Estimated Using } \\
\text { Land Use Typologies }\end{array}$} \\
\hline Subject & Length $/ \mathrm{km}^{2}$ & $\begin{array}{l}\text { Estimated } \\
\text { mean } 2010 \\
\text { Length/km² }\end{array}$ & $\begin{array}{c}\text { Estimated } \\
\text { median } 2010 \\
\text { Length } / \mathrm{km}^{2}\end{array}$ & $\begin{array}{l}\text { Estimated } \\
\text { mean } 2010 \\
\text { Length } / \mathrm{km}^{2}\end{array}$ & $\begin{array}{c}\text { Estimated } \\
\text { median } 2010 \\
\text { Length } / \mathrm{km}^{2}\end{array}$ \\
\hline Maximum & 12479.2 & 21683.6 & 19726.2 & 691351 & 254961 \\
\hline Mean & 1266.87 & 2438.93 & 1024.82 & 4579.88 & 1573.34 \\
\hline Std. Dev. & 1225.54 & 3894.69 & 1586.12 & 14889.9 & 5031.81 \\
\hline Sum & 59929075.52 & 172641820.33 & 72542997.24 & 32330304.62 & 111065222.91 \\
\hline Median & 1019.22 & 842.741 & 292.782 & 744.288 & 244.61 \\
\hline 3rd quartile & 1568.47 & 3090.05 & 1415.11 & 3442.33 & 1239.35 \\
\hline 99.9 Percentile & 10244.1 & 20787.6 & 11607.1 & 186361 & 61655.8 \\
\hline
\end{tabular}

source: authors

values for these categories are $91 \mathrm{~m}$ for rural and 15 $\mathrm{m}$ urban areas respectively.

Using the calculated maps for 2030 and 2050, Fig. 4 displays how the grid requirements will evolve over the next years by providing differences between the estimations in absolute values. These differences are calculated by subtracting a year in the future to one of the previous ones, (eg. 2010 2030, $2010-2050$ and 2030 -2050), which means that negative values represent an increment in grid length and positive values a decrease. Comparing grid length requirements in 2050 with the grid from 2010 , it is easily visible that in areas around the already existing largely urbanized regions, the total grid length per pixel is greatly increased and in all other regions rather decreased. The decrease in grid length requirements can be traced back to a decrease in population within a certain cluster category. Population increments high enough to generate a switch in the population cluster cat- egory but too small to compensate for differences between grid length per person in the population category also induce a decrease in grid length requirements per pixel. This means that in particular cases the switch from rural to urban or urban to city cluster types might lead to a decrease in total required grid length. In practice this would depend on the way that the inhabitants are distributed across the pixels. The cluster type switch assumes that the grid length per person is reduced the more people live in one pixel as their spatial distance to each other shrinks because of a homogenous dispersion of inhabitants. This however is not necessarily the case in reality. Furthermore, rural regions that continue to be classified as a rural region show a decrease in grid length requirements due to overall less people living in the pixels. Based on the selected population density typologies, the total medium voltage grid requirements steadily decrease until 2050, although the population is ex-

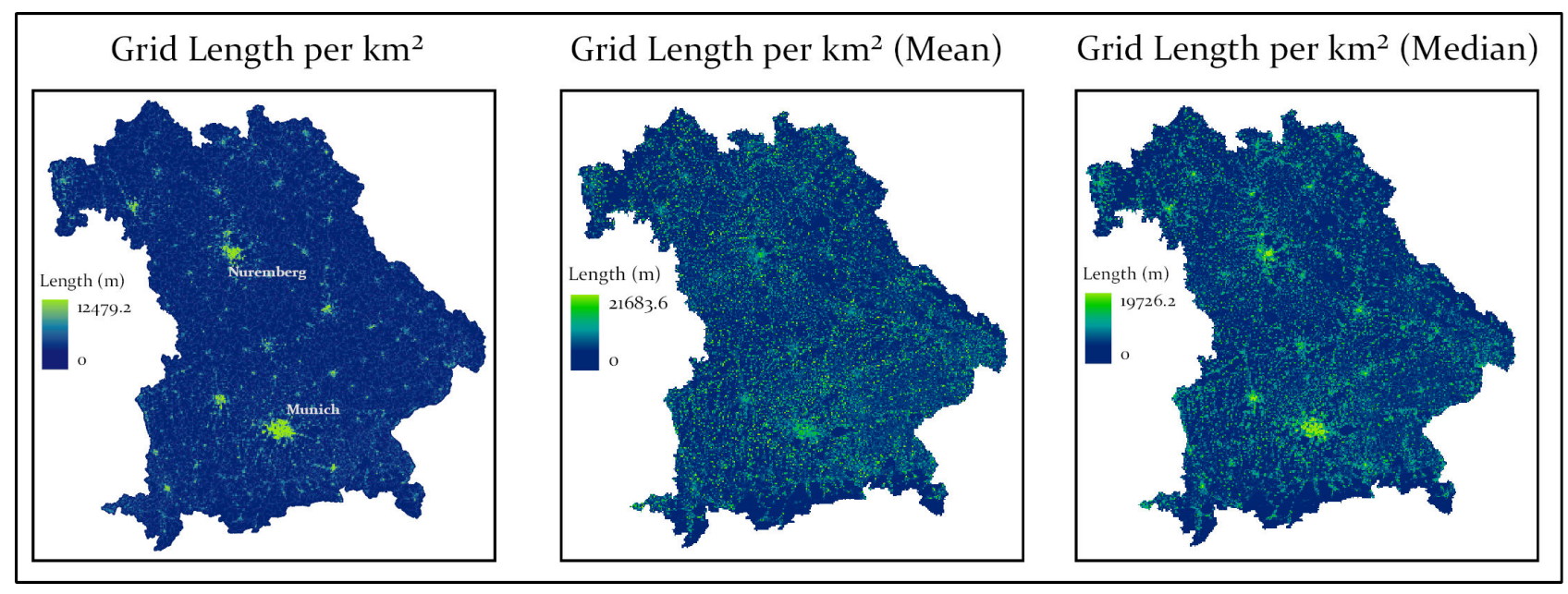

Fig. 3 Medium Voltage Grid Lengths in 2010. Source: authors 
pected to increase until 2030 (see Fig. 1). The expected decrease in total population until 2050 accentuates this diminution.

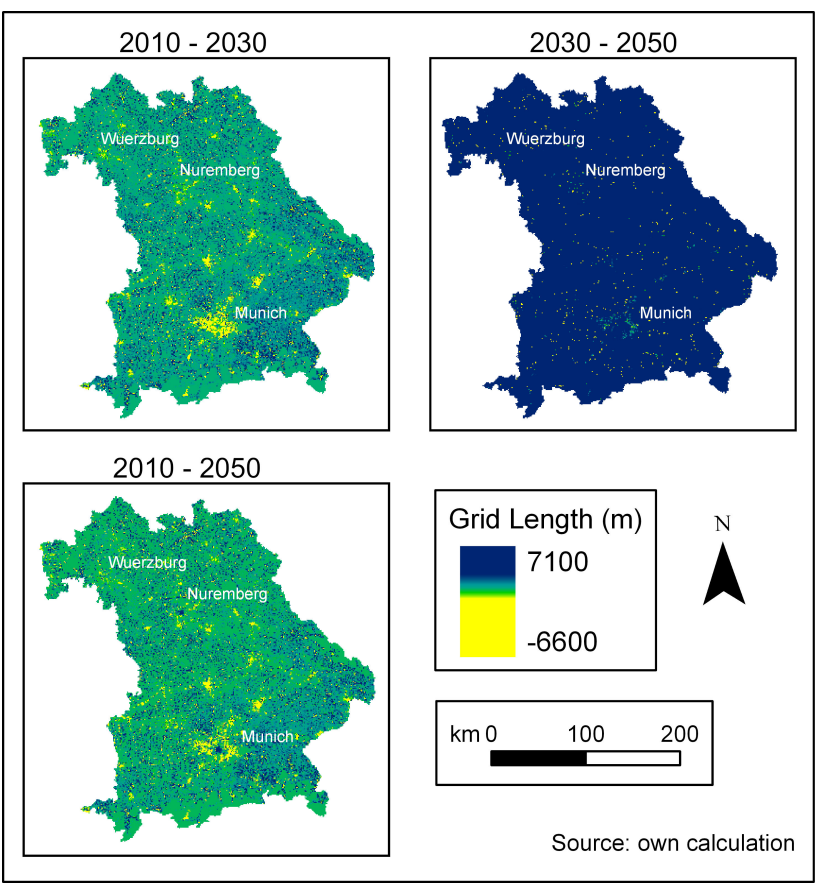

Fig. 4 Medium Voltage Grid Lengths in 2010. Source: authors

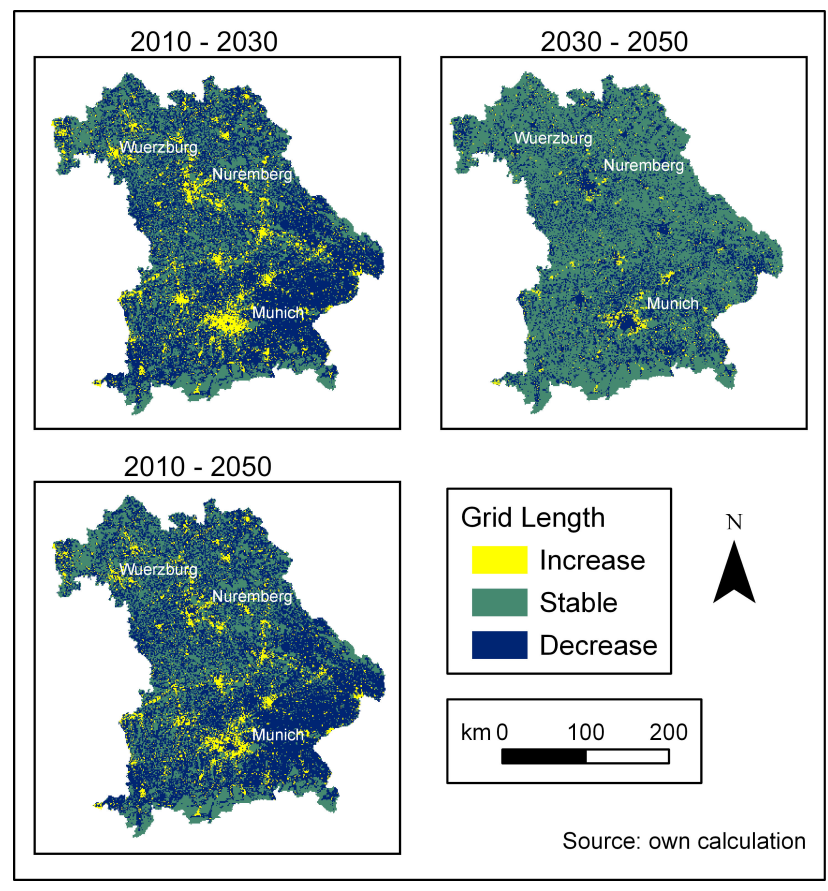

Fig. 5 Medium Voltage Grid Lengths in 2010. Source: authors
The results of the low voltage grid follow the same trends as the medium voltage grid. Fig. 5 shows the results for the low voltage grid reclassified to three categories: decrease (grid length difference larger than $100 \mathrm{~m}$ ), stable (grid length difference between $-100 \mathrm{~m}$ and $100 \mathrm{~m}$ ) and increase (grid length difference smaller than $-100 \mathrm{~m}$ ). The absolute numbers present a very similar picture to Fig. 4 while the reclassified version serves to easily recognize the main trends and to better differentiate large from small changes (see e.g. maps 2030-2050). Between 2010 and 2030 there are increments in grid length for the category city and urban as can be recognized for major cities such as Munich, Nuremberg and Wurzburg. For the period 2030 to 2050 the increments continue mainly in areas categorized as urban, which are predominantly situated in the suburbs of major cities. The depopulation of large rural areas leads to a continuous reduction of grid requirements in most parts of Bavaria. This effect can be observed within the whole forecast period but is stronger pronounced in the period between 2010-2030.

Already today, following the estimations made using median values, the low voltage grid length per person living in a rural region is nearly 13 times higher than for people living in cities. Regarding the medium voltage grid length per person this effect is even more pronounced: the grid length per rural person is here approximately 16 times higher than per person in the city. Such different needs in the electricity infrastructure raises questions on the future handling of the same. The differences in grid length requirements between rural and urban areas will rather increase in the future than decrease. Therefore, when calculating costs for maintenance and expansion per benefiting person, the rural areas have a worse cost-benefit balance than cities. Two important subjects emerge from such results. First, how are the in future even larger cities provided with enough energy, especially when focussing on renewables? Some studies already propose different measures to secure the electricity provision also for large cities. (Waite \& Modi 2016) for example, analyse the possible wind power extension in the United States, trying to cover New York's energy demand at all times by using wind turbines with a baseload of hydropower. Their results show that covering New York's total energy demand will not be possible by focussing solely on wind turbines. Especially the generation locations further away from the city increase losses in electricity during transmission. Another approach is presented by Campana et al. (2016). The authors propose to plan new residen- 
tial urban districts according to the special needs of renewable energy sources and water harvesting possibilities. However, in this scenario special locations need to be reserved only for energy generation sites and the normal density of buildings in urbanized regions cannot be achieved.

The second impact drawn from the results is that considerations need to be taken into account on how rural regions can be supplied with energy, by simultaneously reducing infrastructure costs per person. The well discussed topic of energy supply self-sufficiency could be one potential solution. Selfsufficiency is an approach, which can be applied to different electrical system sizes. It can either include a whole village, a neighbourhood or single family houses but the installed capacity requirements are the key element to be taken into consideration mainly if self-sufficiency is anticipated using only renewable energy sources (Ramirez Camargo et al. 2019). Therefore, especially when planning for the future, the price of maintenance and development of the grid should be compared to the price of promotion and installation measures that would allow a self-sufficient energy supply.

\section{Conclusions}

When not only considering the transition to renewable energies as an important factor for grid extensions and requirements, a whole new picture on needs and possibilities for the power grid emerges. The rising trend to move to cities is not to be neglected. Resulting urbanization and land depopulation streams already have important impacts on today's maintenance needs of the grid. In the particular case of Bavaria, the total grid requirements will decrease by about $30 \%$ for both, the medium and the low voltage grids until 2050. In rather urbanized regions, the grid needs to be prepared for expansions, while in rural regions existent grid infrastructure will become unnecessary. Hot-spots of such changes can be located using the results of the presented methodology. Being based merely on population development, this study presents only a part of the story of the potentially necessary distribution grid development. Future research should integrate this approach and also consider technological changes concerning electric vehicles, the integration of renewables and the possible electrification of heat demands. However, one key element that has to be resolved to gain reliable results, is that data on the official distribution grids covering large areas (e.g. entire states and countries) is publicly available. Such data will not only contribute to refine studies as presented here but will also enable large scale detailed studies of the integration of distributed generation and optimal settlement structures for the development of e.g. energy communities with a very high share of up to $100 \%$ renewable energy supply.

\section{Acknowledgements}

This study was conducted within the framework of the project "CrossEnergy: energy infrastructure - future perspectives for a region in change" (Project number: 036), funded by the European Regional Development Fund and in the frame of the INTERREG V programme between the Federal State of Bavaria (Germany) and the Czech Republic.

\section{References}

Alturki M, Khodaei A, Paaso A, Bahramirad S (2018) Optimization-based distribution grid hosting capacity calculations. Applied Energy 219: 350-360.

p, M. (2004) Landscape change and the urbanization process in Europe. Landscape and Urban Planning 67(1-4), 9-26.

Barbosa A, Jacobs-Crisioni C, Batista e Silva F, Perpiña C, Lavalle $\mathrm{C}$, Baranzelli $\mathrm{C}, \ldots$ Institute for Environment and Sustainability (2015) The reference scenario in the LUISA platform: Updated configuration 2014. Available from: <http://bookshop.europa.eu/uri?target=EUB:NOTICE:LBNA27019:EN:HTML>

Campana PE, Quan SJ, Robbio FI, Lundblad A, Zhang Y, Ma T, Yan J (2016) Spatial Optimization of Residential Urban District-Energy and Water Perspectives. Energy Procedia 88: 38-43.

Child M, Nordling A, Breyer C (2018) The Impacts of High V2G Participation in a 100\% Renewable Åland Energy System. Energies 11(9): 2206.

Dijkstra L, Poelman H (2014) A harmonised definition of cities and rural areas: The new degree of urbanisation. 28.

GDAL Development Team (2016) GDAL-Geospatial Data Abstraction Library. Available from:<http://www.gdal.org>

Guntaka R, Myler HR (2014) Regression and kriging analysis for grid power factor estimation. Journal of Electrical Systems and Information Technology 1(3): 223-233.

Hess D, Wetzel M, Cao K-K (2018) Representing node-internal transmission and distribution grids in energy system models. Renewable Energy 119: 874-890.

Keirstead J, Jennings M, Sivakumar A (2012) A review of urban energy system models: Approaches, challenges and opportunities. Renewable and Sustainable Energy Reviews 16(6): 3847-3866.

Kruskal JB (1956) On the Shortest Spanning Subtree of a Graph and the Traveling Salesman Problem. Proceedings of the American Mathematical Society 7(1): 48-50.

Marczinkowski HM, Østergaard PA (2018) Residential versus communal combination of photovoltaic and battery in smart energy systems. Energy 152: 466-475.

Neteler M, Mitasova H (2008) Open source GIS: A grass GIS approach. Springer, New York. 


\section{S sciendo}

Öko-Institut e.V. Institute für angewandte Ökologie (2019). Energiewende: Geschichte, aktuelle Situation, Zukunft. Available from: <http://www.energiewende.de/start>

OpenStreetMap (2018). OpenStreetMap- Deutschland. Available from: <https://www.openstreetmap.de>

Østergaard PA, Andersen FM, Kwon PS (2015) Energy systems scenario modelling and long term forecasting of hourly electricity demand. International Journal of Sustainable Energy Planning and Management 95-112.

Prasomthong J, Ongsakul W, Meyer J (2014) Optimal placement of vehicle-to-grid charging station in distribution system using Particle Swarm Optimization with time varying acceleration coefficient. 2014 International Conference and Utility Exhibition on Green Energy for Sustainable Development (ICUE), pp. 1-8.

Ramirez Camargo L, Nitsch F, Gruber K, Valdes J, Wuth J Dorner W (2019) Potential Analysis of Hybrid Renewable Energy Systems for Self-Sufficient Residential Use in
Germany and the Czech Republic. Energies, 12(21), 4185. https://doi.org/10.3390/en12214185

Ramirez Camargo L, Stoeglehner G (2018) Spatiotemporal modelling for integrated spatial and energy planning. Energy, Sustainability and Society 8(1).

Short TA (2004) Electric power distribution handbook. CRC Press, Boca Raton.

Stiens G (1986) On the future of settlement structures. Futures 18(1): 24-39.

Tafarte P, Eichhorn M, Thrän D (2019) Capacity Expansion Pathways for a Wind and Solar Based Power Supply and the Impact of Advanced Technology-A Case Study for Germany. Energies 12(2): 324.

Waite M, Modi V (2016) Modeling wind power curtailment with increased capacity in a regional electricity grid supplying a dense urban demand. Applied Energy 183: 299-317. 